\title{
Abschreiben von Übungsaufgaben in traditionellen und innovativen Mathematikvorlesungen
}

Michael Liebendörfer und Robin Göller

Die Abgabe von Übungsaufgaben ist in den ersten Jahren des Mathematikstudiums an den meisten deutschen Universitäten der Standard. Einige Studierende erarbeiten sich die Lösungen aber nicht selbst, sondern schreiben sie aus fremden Quellen ab. Den Lehrenden ist das durchaus bekannt. Aus hochschuldidaktischer Perspektive ist dieses Thema spannend und praxisrelevant, allerdings fast vollkommen unerforscht. Wir haben versucht, das Phänomen des Abschreibens in einer traditionell gehaltenen Vorlesung durch die Analyse von Interviews mit einzelnen Studierenden sowie durch die Analyse von Daten aus Fragebogenstudien etwas konkreter zu umreißen [4]. In diesem Artikel wollen wir die zentralen Befunde noch einmal kurz benennen. Anschließend wird die Situation für innovative Vorlesungen mit veränderten Inhalten und Übungsaufgaben anhand einer Untersuchung analysiert, die wir in [5] bereits skizziert haben. Der Vergleich beider Formen zeigt, dass im neuen Format deutlich weniger abgeschrieben wird. Schlussendlich diskutieren wir die Bedeutung der Befunde für die Lehre.

\section{Abschreiben in traditionellen Vorlesungen}

In der Literatur finden sich zwei Untersuchungen von Studierenden aus der Veranstaltung Analysis I an der Universität Kiel, die zur Semestermitte durchgeführt wurden $[6,7]$. Studierende sollten dabei einschätzen, ob sie

- ihre Aufgabenlösungen einfach abschreiben, ohne die Lösungen nachzuvollziehen,

- versuchen Lösungen anderer nachzuvollziehen, aber nur selten eigene Erklärungen oder Verbesserungen geben,

- oft nicht selbst auf die Lösung kommen, aber diese verbessern und/oder sich und Kommilitonen erklären,

- ihre Aufgaben selbst lösen.

Dem ersten Typ hatte sich niemand zugeordnet, dem zweiten Typ etwa ein Drittel der Studierenden, dem dritten Typ etwas mehr als die Hälfte und dem vierten Typ etwa ein Siebtel. Die Studierenden des letzten Typs hatten besonders gute Schulleistungen und sind auch selbst von ihrer Fähigkeit überzeugt. In Studie [6] wiesen Studierende der unterschiedlichen Typen in der Klausur sehr unterschiedliche Bestehensquoten auf: der nachvollziehende Typ erreichte nur gut $10 \%$, die Selbsterklärer lagen bei $40 \%$ und die Selbstlöser kamen auf etwa $75 \%$. Diese Trennung ist bemerkenswert, da sie schon zur Semestermitte möglich ist, also zu einem Zeitpunkt an dem man noch intervenieren könnte. Es deutet sich zudem an, dass die große Mehrheit der Studierenden weder einfach abschreibt, noch die Aufgaben selbst löst.

\section{I.I Was ist Abschreiben?}

Anhand von Interviews mit 21 Erstsemester-Studierenden der Universität Kassel (Fachstudium Mathematik, Lehramt Mathematik und Fachstudium Physik) haben wir in [4] herausgestellt, dass die Unterschiede zwischen Abschreiben und selbständigem Lösen eher graduell verlaufen.

Der Leser möge selbst bewerten, welche der folgenden, in den Interviews genannten Aktivitäten für ihn eine unzulässige Form des Abschreibens darstellen:

- sich Tipps in Internet-Foren holen,

- gemeinsame Lösungen mit Kommilitonen entwickeln,

- dabei eine Idee verwenden, die andere eingebracht haben,

- eine gemeinsam erarbeitete Lösung aufschreiben, zu der man trotz reichlichen Bemühens selbst nichts beitragen konnte,

- eine gemeinsam erarbeitete Lösung aufschreiben, zu der man aufgrund geringen Bemühens nichts beitragen konnte,

- eine fremde Lösung betrachten, beiseitelegen und eine eigene Lösung mit den gleichen Argumenten aufschreiben,

- eine fremde Lösung betrachten und in der Notation variieren, damit sie nicht identisch aussieht,

- eine fremde Lösung zeichenweise kopieren.

Wenn man bedenkt, dass Studierende durchaus lernen sollen, in die Literatur zu schauen und mit Kommilitonen zusammenzuarbeiten, kann man kaum eine scharfe Trennlinie zwischen Abschreiben und eigenständigem Arbeiten ziehen. Anstelle der Frage, ob Studierende entweder abschreiben oder eigenständig arbeiten, scheint die Frage passender, wie sehr Studierende eigenständig arbeiten.

\section{I.2 Wie und warum wird abgeschrieben?}

Die Quellen für fremde Lösungen sind sehr vielfältig. Verwendet werden klassische Medien wie Lehrbücher, Skripte, etc. Der Einsatz digitaler Technologien schafft dabei eine große Verfügbarkeit von Lösungen. Die Verfügbarkeit alleine begründet aber das Abschreiben noch nicht. 
[...] wir versuchen es schon selber mal und vergleichen dann halt manchmal mit [der Abschreibequelle]. Aber angenommen es ist dann $8 \mathrm{Uhr},[\ldots]$ dann sagen wir [...] in I, 2 Stunden müssen wir ja jetzt alle weg und dann geht es halt: Und der Rest wird jetzt halt abgeschrieben.

Unser Eindruck aus den Interviews war: Obwohl Quellen zum Abschreiben leicht verfügbar sind, versuchen die Studierenden zunächst, ihre Aufgaben selbst zu lösen. Bei mangelndem Erfolg, erhöhter Frustration und Zeitmangel, steigt die Bereitschaft, Aufgaben abzuschreiben.

Wichtige Gründe für das Abschreiben sind die Verfügbarkeit von Aufgabenlösungen, eine geringe Bereitschaft, sich anzustrengen und Effizienzüberlegungen. Als Hauptgrund sehen wir jedoch die fachliche Überforderung mit den Aufgaben.

Also, ich habe auch immer mal zwischendrin wieder probiert, eine Aufgabe selber zu machen. [...] Und wenn ich mit JEDER Aufgabe, die ich selber genau auf dem Weg probiert habe, echt auf die Nase falle / weil ich brauche ja diese scheiß $50 \%$ um überhaupt die Zulassung zu bekommen, ja dann lasse ich es doch irgendwann.

Das Abschreiben ist dabei für alle interviewten Studierenden eine mögliche Option für das Erreichen der Klausurzulassung - auch für die, die gar nicht abgeschrieben hatten. Sie wird aber als sehr unangenehm, demotivierend und nicht verträglich mit dem eigenen Selbstbild empfunden.

\section{I.3 Wer schreibt $a b$ - und in welchem Umfang?}

Ebenfalls in [4] haben wir die Daten einer Fragebogenstudie unter Studierenden aus der Linearen Algebra $I$ an der Universität Kassel präsentiert. Für die Erhebung haben wir eine Formulierung genutzt, die sich nur auf das Abschreiben von Kommilitonen und nicht aus Büchern oder Internetquellen bezieht und ausschließen soll, dass die Studierenden am Prozess der Aufgabenlösung beteiligt waren: „Beim Bearbeiten von Übungsblättern habe ich dieses Semester fertige Lösungen von anderen Studenten übernommen“. Daneben wurden einige Fragen gestellt, etwa ob man sich mit den Aufgaben überfordert fühlt oder wie hoch die eigene Anstrengungsbereitschaft ist.

Abbildung I zeigt, dass - wie in den Studien [6, 7] - auch hier nur etwa ein Siebtel der Studierenden vollständig ohne Abschreiben auskommt. Bedenklich ist dabei nicht nur, dass viele Studierende überhaupt abschreiben, sondern, dass die Mehrheit von ihnen eher oft als selten abschreibt. Die Lehramtsstudierenden schreiben dabei etwas mehr ab als die Fachstudierenden, der Unterschied ist aber statistisch nicht klar abgesichert (zweiseitiger $t$ Test, $p=0.085)$. Zwischen den Geschlechtern zeigt sich kein Unterschied.

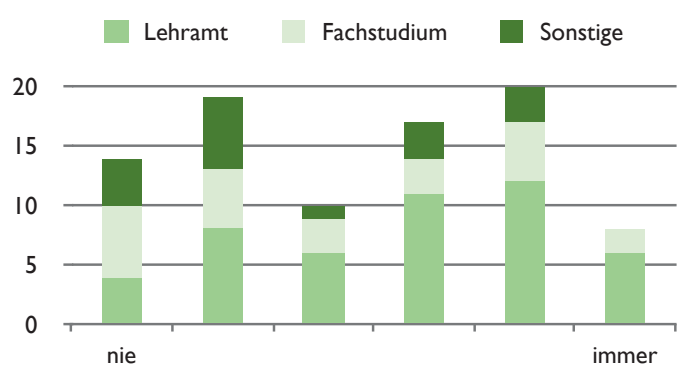

Abbildung I. Absolute Häufigkeiten der Antworten auf die Frage: „Beim Bearbeiten von Übungsblättern habe ich dieses Semester fertige Lösungen von anderen Studenten übernommen.“, $N=88$

Die statistische Analyse zeigt außerdem eindeutige Korrelationen, die unseren Eindruck aus den Interviews bestätigt haben: Studierende, die öfter abschreiben sind meistens mit den Aufgabenstellungen überfordert und haben ein geringeres Vertrauen in die eigene Fähigkeit, die Aufgaben zu lösen. Sie geben an, bei den Übungsaufgaben nicht zu wissen, was sie tun sollen, sind der Meinung, dass sie die Aufgaben nicht selbständig lösen können, und dass ihnen bei eigenen Lösungen zu viele Punkte abgezogen werden. Außerdem haben sie eher eine geringere Anstrengungsbereitschaft und schätzen das selbständige Bearbeiten der Aufgaben als ineffektiv ein. Schließlich kommt Abschreiben häufiger bei Studierenden vor, die in Gruppen arbeiten.

\section{Abschreiben in innovativen Veranstaltungen}

Die bisher präsentierten Daten stammen überwiegend aus dem Wintersemester 2012/20I3. Mittlerweile ist die Studieneingangsphase an der Universität Kassel umstrukturiert worden. Insbesondere wurde die neue Veranstaltung Grundlagen der Mathematik eingeführt, die zum Ziel hat, in die Sprache und die Methoden der Mathematik einzuführen. Dazu sollen grundlegende Beweistechniken und Problemlösestrategien eingeübt werden und so das Verstehen und Formulieren von Mathematik vermittelt werden. Im Wintersemester 2015/2016 folgte diese Vorlesung inhaltlich zu großen Teilen dem von Daniel Grieser in Oldenburg entwickelten und in seinem Buch [3] ausgeführten Ansatz. In Oldenburg ist dieser Ansatz Grundlage des Moduls Mathematisches Problemlösen und Beweisen. Es soll einen kreativen, problemorientierten Zugang zur Mathematik bieten, der in Studierenden Freude an der Beweisführung, Neugierde auf mathematische Theorien und das Gefühl, Mathematik verstehen zu können, weckt und gleichzeitig anspruchsvolle Mathematik behandelt. Inhaltlich werden darin anhand elementarer, intuitiv leicht zugänglicher Themen explizit verschiedene Beweis- und Problemlösestrategien herausgestellt.

In Kassel wurde zudem mit Blick auf diese Überforderungssymptome versucht, die Zugänglichkeit der Aufgaben zu erhöhen. So wurden strategische Ansätze, 
die sonst oft in der abgegebenen Lösung nicht mehr auftauchen, wie etwa das Betrachten von Beispielen, Spezialfällen oder Skizzen, oder das Formulieren von Vermutungen und Zwischenzielen, als Teilaufgaben formuliert, um sie sichtbar und honorierbar werden zu lassen. Außerdem wurde besonderer Wert darauf gelegt, dass beim Korrigieren der Aufgaben eigene Lösungsansätze honoriert wurden.

\section{I Ergebnisse zum Abschreiben}

Auch in diesen innovativen Veranstaltungen haben wir die Frage gestellt, inwieweit die Studierenden beim Lösen der Übungsaufgaben fertige Lösungen von anderen Studierenden übernommen haben.

Die Daten (siehe Abbildung 2) unterscheiden sich erheblich von den Befunden der traditionellen Vorlesungen. In Oldenburg schrieb ein gutes Viertel nie ab, in Kassel sogar ein gutes Drittel der Studierenden. Nur noch $18 \%$ der Studierenden beider Veranstaltungen gaben an, eher oft als selten abzuschreiben und es fand sich nur noch eine Person, die angab, immer abzuschreiben. Daneben zeigt sich erneut die Tendenz, dass die Fachstudierenden weniger abschreiben, die - nicht in Kassel, aber in Oldenburg - signifikant ist (zweiseitiger $t$-Test, $p<0.001$ ). Hierbei muss jedoch angemerkt werden, dass die Veranstaltung in Oldenburg als Wahlfach im Fachstudium vor allem von Studierenden im dritten Fachsemester besucht wird.

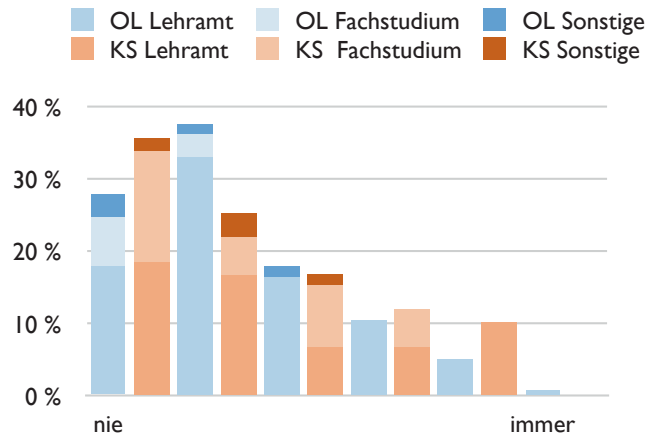

Abbildung 2. Antworten auf die Frage: „Beim Bearbeiten von Übungsblättern habe ich dieses Semester fertige Lösungen von anderen Studenten übernommen.", $N=133$ in Oldenburg und $N=59$ in Kassel

\section{Diskussion}

Bereits in Fallstudien, die Ende der 1970er-Jahre entstanden, wurde gezeigt, dass Übungsblätter abgeschrieben werden [2]. Auch in anderen Studienfächern zeigt eine Untersuchung [8], dass Studierende Hausaufgaben abschreiben, bei Hausarbeiten plagiieren oder Daten aus Experimenten manipulieren. Aber das Ausmaß des Abschreibens in der traditionellen Linearen Algebra ist doch sehr hoch. Die Tatsache, dass nur etwa ein Siebtel der
Studierenden die Aufgaben in der angedachten Form bearbeitet, sollte uns zum Nachdenken anregen. Dabei muss bedacht werden, dass die Formulierung unserer Frage nach dem Abschreiben noch kein Abschreiben aus Büchern oder Internet-Quellen beinhaltet und auch kein Abschreiben von Lösungen, die nicht ,fertig“ übernommen wurden. Die Arbeiten [6,7] deuten außerdem an, dass sich diese Befunde zahlenmäßig verallgemeinern lassen. Insofern ist überraschend, wie wenig diese Problematik in Diskussionen und Modellen guter Lehre thematisiert wird.

Dabei muss das Abschreiben aus unserer Sicht vor allem als Symptom einer fachlichen Überforderung angesehen werden. Der Hauptgrund für die deutlich niedrigeren Abschreibequoten in den beiden innovativen Vorlesungen ist unserer Meinung nach die deutlich geringere Überforderung der Studierenden mit den Übungsaufgaben der innovativen Vorlesungen [5]. Natürlich spielen aber auch Faktoren wie Anstrengungsbereitschaft, Selbstvertrauen und Gruppenarbeit eine Rolle.

Welche Konsequenzen sich daraus für die Lehre ergeben, ist zunächst unklar. Ein Absenken des Niveaus ist für die meisten Vorlesungen wohl keine Option. Um das Abschreiben zu verhindern, wäre es viel einfacher, die Abgabepflicht abzuschaffen. Dies ist an einigen Universitäten durch die Neuakkreditierung von Studiengängen schon der Fall. Die Auswirkungen müssen abgewartet werden. Allerdings werden die Übungsblätter gestellt, um Studierende bei der Beschäftigung mit dem Stoff der Vorlesungen zu unterstützen. Die verpflichtende Abgabe liefert dafür extrinsische Motivation. Insofern ist bemerkenswert, dass in einer Untersuchung aus England Studierende, die keine verpflichtenden Übungsaufgaben hatten, sich solchen Druck von außen gewünscht haben [I]. Auch die von uns interviewten Studierenden schätzten diesen äußeren Druck in der Regel als durchaus positiv für ihre Motivation und ihr Lernen ein.

\section{I Abschreiben und Lernen}

Wer Lösungen abschreibt, ohne sich mit den Aufgaben ausführlich zu beschäftigen, verringert seine Chancen, die Lernziele zu erreichen. Allerdings haben wir in den Interviews gesehen, dass auch diejenigen, die abschreiben, sich mit den Aufgaben beschäftigen. Im schlimmsten Fall hatten Studierende eigene, korrekte Lösungen entwickelt, ihnen aber nicht vertraut und eine falsche, fremde Lösung abgeschrieben und eingereicht. Diese Situation ist unglücklich, mit Blick auf den Lernerfolg aber weniger schlimm. Studierende, die einen Beweis zumindest ausführlich prüfen, bevor sie ihn abschreiben, beschäftigen sich ebenfalls mit den Inhalten und lernen daraus.

\subsection{Abschreiben als Element des Heterogenitätsmanagements}

Abschreiben, das zeigen die Interviews, ist keine frei gewählte Strategie, sondern eher die Notlösung. Neben 
dem Ausdruck einer Überforderung mit den Aufgaben ist es auch eine praktische Möglichkeit, die Anforderungen für den Modulerfolg trotzdem zu erfüllen. In einer etwas verwegenen Interpretation könnte man das Abschreiben von Übungsblättern als festen Bestandteil des Mathematikstudiums bezeichnen, der eine spezifische Funktion erfüllt: Die Übungsaufgaben sollen einen zu erreichenden Standard setzen und für alle Studierenden einen Lernanreiz bieten, also insbesondere auch für das Siebtel, das sie regelmäßig eigenständig lösen kann. Dafür müssen die Aufgaben herausfordernd und ihre eigenständige Bearbeitung nicht einfach zu umgehen sein. Das Abschreiben erfüllt dann die Funktion, dass auch leistungsschwächere Studierende zumindest bis zur Klausur dabeibleiben können. Die Hürde des Verbotenen bewirkt, dass die Handlungsoption des Abschreibens fast nur von denen genutzt wird, die die Aufgaben ohnehin nicht selbst lösen könnten. Abschreiben ist in dieser Sicht ein Element des Heterogenitätsmanagements. So könnte sich erklären, dass das Abschreiben von Übungsblättern nach unserem Eindruck oftmals toleriert ist.

\subsection{Praktische Überlegungen}

Auch wenn das Abschreiben somit für die Regulierung der formalen Bedingungen eine Funktion hat, werden dadurch die inhaltlichen Schwierigkeiten nicht gelöst. Vielmehr ist zu vermuten, dass das Abschreiben die Heterogenität im Kenntnisstand der Studierenden weiter verstärkt. Daneben sollten aus unserer Sicht die negativen Auswirkungen auf die Motivation der Studierenden nicht unterschätzt werden.

Im derzeitigen System ist unklar, was Studierende, die die Aufgaben nicht selbständig lösen können, tun sollten. Nicht abzuschreiben, würde wohl für viele den Studienabbruch bedeuten. Handlungsmöglichkeiten für die Universitäten haben sich dagegen in den innovativen Veranstaltungen angedeutet. Das Abschreiben wurde zwar auch dort nicht vollständig verhindert, jedoch deutlich reduziert. Wichtig dafür war unserer Meinung nach die Zugänglichkeit der Aufgaben, was wohl auch an den eher elementaren Inhalten lag. Grundsätzlich sollte sich aber die Zugänglichkeit von Aufgaben jeder Grundlagenveranstaltung des Mathematikstudiums erhöhen lassen, indem man etwa das Betrachten von Beispielen, Spezialfällen oder Skizzen, oder das Formulieren von Vermutungen und Zwischenzielen, den eigentlichen Aufgaben als Teilaufgaben voranstellt. Wenn man sich darauf beschränkt, müsste man das Niveau der Aufgaben nicht ändern.

Natürlich wird man bei der stärkeren Strukturierung von Übungsaufgaben abwägen müssen, wie weit man dabei geht. In den höheren Semestern sollten Studierende gelernt haben, selbst einzuschätzen, wann ihnen ein Beispiel oder eine Skizze hilfreich ist. Will man jedoch den heterogenen Eingangsvoraussetzungen der Studierenden wirklich gerecht werden, wird es nötig sein, Aufgaben zu entwickeln, die möglichst vielen Studierenden das selbständige Bearbeiten, zumindest von Teilaufgaben ermöglichen und gleichzeitig alle Studierenden fordern.

\section{Literatur}

[I] Margaret Brown and Sheila Macrae. Full report of research activities and results: students' experiences of undergraduate mathematics (Reference number: R000238564). Technical report, King's College London Department of Education and Professional Studies, London, UK, 2005.

[2] Kristin Gisbert. Frauenuntypische Bildungsbiographien: DiplomMathematikerinnen. P. Lang, Frankfurt am Main; New York, 1995.

[3] Daniel Grieser. Mathematisches Problemlösen und Beweisen. Springer Fachmedien Wiesbaden, Wiesbaden, 2013.

[4] Michael Liebendörfer und Robin Göller. Abschreiben - ein Problem in mathematischen Lehrveranstaltungen? In Walther Paravicini and Jörn Schnieder, editors, Hanse-Kolloquium zur Hochschuldidaktik der Mathematik 2014 Beiträge zum gleichnamigen Symposium am 7. \& 8. November 2014 an der Westfälischen Wilhelms-Universität Münster, Seite I19-14I. WTM-Verlag für wissenschaftliche Texte und Medien, Münster, 2016.

[5] Robin Göller und Michael Liebendörfer. Eine alternative Einstiegsvorlesung in die Fachmathematik - Konzept und Auswirkungen. In BzMU 2016, Heidelberg, 2016. (im Druck).

[6] Stefanie Rach and Aiso Heinze. Welche Studierenden sind im ersten Semester erfolgreich? Journal für Mathematik-Didaktik, 34(I): I $21-147,2013$.

[7] Stefanie Rach and Aiso Heinze. The Transition from School to University in Mathematics: Which Influence Do School-Related Variables Have? International Journal of Science and Mathematics Education, 2016. doi: 10.1007/s 10763-016-9744-8

[8] Sebastian Sattler, Floris van Veen, Andrea Schulze, and Madlen Preuß. FAIRUSE - Fehlverhalten und Betrug bei der Erbringung von Studienleistungen: Individuelle und organisatorischstrukturelle Bedingungen. Technical report, Fakultät für Soziologie der Universität Bielefeld, Bielefeld, 2013.

Michael Liebendörfer, Institut für Didaktik der Mathematik und Physik, Leibniz Universität Hannover, Welfengarten I, 30167 Hannover. liebendoerfer@khdm.de

Robin Göller, Institut für Mathematik, Universität Kassel, HeinrichPlett-Straße 40, 34l09 Kassel. goeller@khdm.de

Michael Liebendörfer hat Mathematik in Konstanz und Tübingen studiert. Seit 2010 ist er Wissenschaftlicher Mitarbeiter bei Prof. Dr. Reinhard Hochmuth, aktuell an der Leibniz Universität Hannover. Forschungsschwerpunkt: Motivationsentwicklung im Mathematikstudium.

Robin Göller hat Mathematik an der Universität Konstanz studiert. Seit 2012 ist er Wissenschaftlicher Mitarbeiter bei Prof. Dr. Hans-Georg Rück an der Universität Kassel. Forschungsschwerpunkt: Lernstrategien und selbstreguliertes Lernen von Mathematikstudierenden.

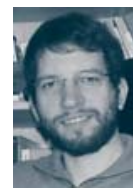

Robin Göller und Michael Liebendörfer sind Mitarbeiter im Kompetenzzentrum Hochschuldidaktik Mathematik (khdm). 